\title{
Symmetry and Duality in Lévy Markets*
}

\author{
José Fajardo ${ }^{\dagger}$ and Ernesto Mordecki ${ }^{\ddagger}$ \\ February 16, 2006
}

\begin{abstract}
The aim of this paper is to introduce the notion of symmetry in a Lévy market. This notion appears as a particular case of a general known relation between prices of put and call options, of both the European and the American type, that is also reviewed in the paper, and that we call put-call duality. Symmetric Lévy markets have the distinctive feature of producing symmetric smile curves, in the log of strike/futures prices.

Put-Call Duality is obtained as a consequence of a change of the risk neutral probability measure through Girsanov's Theorem, when considering the discounted and reinvested stock price as the numeraire. Symmetry is defined when a certain law before and after the change of measure through Girsanov's Theorem coincides. A parameter characterizing the departure from symmetry is introduced, and a necessary and sufficient condition for symmetry to hold is obtained, in terms of the jump measure of the Lévy process, answering a question raised by Carr and Chesney (1996). Some empirical evidence is shown, supporting that in general markets are not symmetric.
\end{abstract}

Keywords: Lévy processes, Girsanov Theorem, Smile, Numeraire, Duality, Symmetry.

${ }^{*}$ We thank comments from two anonymous referees, the conference participants at The 2004 North American Winter Meeting of the Econometric Society and III World Congress of The Bachelier Finance Society, and Álvaro Cartea for helpful discussion. The usual disclaimer applies. J. Fajardo also thanks CNPq and PRONEX E-26/171.193/2003 for financial support.

${ }^{\dagger}$ IBMEC Business School, Rio de Janeiro - Brazil, e-mail: pepe@ibmecrj.br

${ }_{\ddagger}^{\ddagger}$ Centro de Matemática, Facultad de Ciencias, Universidad de la República, Montevideo. Uruguay. e-mail: mordecki@cmat.edu.uy. 


\section{Introduction}

Change of numeraire - Change of probability measure is a well known technique in derivative pricing. Geman et al. (1995) observe that any positive price process can be used as a numeraire, establishing the consequences of the corresponding change of probability measure in a market model of continuous semimartingales; and apply their results to several derivative pricing situations, unifying and extending known results.

After other developments, Schroder (1999) uses the reinvested and discounted stock price process as a numeraire, in a semimartingale model, extending an initial foreign currency result due to Grabbe (1983). He obtains a call-put relationship that here we name duality. In this denomination we follow, on one side, the classical notion of dual Lévy process to refer to the opposite of a given Lévy process (see Bertoin (1996)) that appears as the driving process after the change of numeraire and, on the other, Shepp and Shiryaev (1994) and Kramkov and Mordecki (1994) that name as dual measure the probability measure defined by Girsanov Theorem when this numeraire is used as the density process. We go further and refer to the dual market and dual stock price process to identify the market model under this new probability measure, and the resulting stock price process. A review of the notion of put-call duality in the framework of Itô processes can be found in Detemple (2001). Our results were announced in Fajardo and Mordecki (2003). Related results can be found in Eberlein and Papantaleon (2005). In Fajardo and Mordecki (2005) we consider similar problems for time-changed Lévy processes.

In the presented paper we derive the put-call duality in the framework of a Lévy driven stock market model. On one side our results are a particular case of the ones obtained by Schroder (Corollary 1, 1999) but, on the other, the nature of Lévy processes, besides giving the possibility of an elementary treatment (without the use of semimartingale calculus) give a precise description of the distribution of the dual stock price process in the dual market. This is done in Section 2.

In Section 3 we introduce the notion of a symmetric Lévy market, to distinguish the situation when the discounted and reinvested stock in the original risk neutral market, and the corresponding dual price process under the dual measure have the same probability law. Put-Call symmetry was investigated by Carr and Chesney (1996) for diffusion models, generalizing the symmetry observed in the Black-Scholes model. We observe that the volatility smile 
curve constructed from option prices given by a symmetric Lévy market is a symmetric curve when plotted as a function of the logarithm of the ratio between the strike and the futures price.

Our law characterization in the dual market gives as a consequence necessary and sufficient conditions for symmetry to hold, expressed in terms of the Lévy jump measure of the original process. This generalizes a result by Bates (1997) obtained in the Merton's (1976) jump diffusion model.

Furthermore, in Section 4, assuming that the Lévy jump measure corresponding to the driving process has a specific particular form, that is used in practically all parametric models found in practice, we identify a "symmetry" parameter, and explore whether Lévy market models reported in the literature are symmetric. Our conclusion is that in general, symmetry does not hold.

We give some conclusions and propose some topics for further research in Section 5.

\section{Lévy processes and Duality}

Consider a real valued stochastic process $X=\left\{X_{t}\right\}_{t \geq 0}$, defined on a stochastic basis $\mathcal{B}=\left(\Omega, \mathcal{F}, \mathbf{F}=\left(\mathcal{F}_{t}\right)_{t \geq 0}, \mathbf{Q}\right)$, being càdlàg, adapted, satisfying $X_{0}=0$, and such that for $0 \leq s<t$ the random variable $X_{t}-X_{s}$ is independent of the $\sigma$-field $\mathcal{F}_{s}$, with a distribution that only depends on the difference $t-s$. Assume also that the stochastic basis $\mathcal{B}$ satisfies the usual conditions (see [20]). The process $X$ is a Lévy process, and is also called a process with stationary independent increments (PIIS). For general reference on Lévy processes see [20], [36], [2], [28]. For Lévy process in Finance see Boyarchenko and Levendorskii (2002), Schoutens (2003) and Cont and Tankov (2004).

In order to characterize the law of $X$ under $\mathbf{Q}$, consider, for $q \in \mathbb{R}$ the Lévy-Khintchine formula, that states

$$
\mathbf{E} e^{i q X_{t}}=\exp \left\{t\left[i a q-\frac{1}{2} \sigma^{2} q^{2}+\int_{\mathbb{R}}\left(e^{i q y}-1-i q h(y)\right) \Pi(d y)\right]\right\},
$$

with

$$
h(y)=y \mathbf{1}_{\{|y|<1\}}
$$

a fixed truncation function, $a$ and $\sigma \geq 0$ real constants, and $\Pi$ a positive measure on $\mathbb{R} \backslash\{0\}$ such that $\int\left(1 \wedge y^{2}\right) \Pi(d y)<+\infty$, called the Lévy mea- 
sure. The triplet $\left(a, \sigma^{2}, \Pi\right)$ is the characteristic triplet of the process, and completely determines its law.

Consider the set

$$
\mathbb{C}_{0}=\left\{z=p+i q \in \mathbb{C}: \int_{\{|y|>1\}} e^{p y} \Pi(d y)<\infty\right\} .
$$

The set $\mathbb{C}_{0}$ is a vertical strip in the complex plane. For arbitrary Lévy process this set contains the line $z=i q(q \in \mathbb{R})$, and consists of all complex numbers $z=p+i q$ such that $\mathbf{E} e^{p X_{t}}<\infty$ for some $t>0$.

Due to the martingale condition in financial market models, we assume that $\mathbf{E} e^{X_{1}}<\infty$, concluding that $\mathbb{C}_{0}$ contains the strip $0 \leq \operatorname{Re}(z)=p \leq 1$. Furthermore, if $z \in \mathbb{C}_{0}$, we can define the characteristic exponent of the process $X$, by

$$
\psi(z)=a z+\frac{1}{2} \sigma^{2} z^{2}+\int_{\mathbb{R}}\left(e^{z y}-1-z h(y)\right) \Pi(d y)
$$

this function $\psi$ is also called the cumulant of $X$, having $\mathbf{E} e^{z X_{t}}<\infty$ for all $t \geq 0$, and $\mathbf{E} e^{z X_{t}}=e^{t \psi(z)}$. The finiteness of this expectations follows from Theorem 25.3 in [28]. Formula (3) reduces to formula (1) when $\operatorname{Re}(z)=0$.

\subsection{Lévy market}

By a Lévy market we mean a model of a financial market with two assets: a deterministic savings account $B=\left\{B_{t}\right\}_{t \geq 0}$, with

$$
B_{t}=e^{r t}, \quad r \geq 0,
$$

where we take $B_{0}=1$ for simplicity, and a stock $S=\left\{S_{t}\right\}_{t \geq 0}$, with random evolution modelled by

$$
S_{t}=S_{0} e^{X_{t}}, \quad S_{0}=e^{x}>0,
$$

where $X=\left\{X_{t}\right\}_{t \geq 0}$ is a Lévy process.

In this model we assume that the stock pays dividends, with constant rate $\delta \geq 0$, and that the given probability measure $\mathbf{Q}$ is the chosen equivalent martingale measure. In other words, prices are computed as expectations with respect to $\mathbf{Q}$, and the discounted and reinvested process $\left\{e^{-(r-\delta) t} S_{t}\right\}$ is a Q-martingale. 
In terms of the characteristic exponent of the process this means that

$$
\psi(1)=r-\delta,
$$

based on the fact, that $\mathbf{E} e^{-(r-\delta) t+X_{t}}=e^{-t(r-\delta+\psi(1))}=1$, and condition (5) can also be formulated in terms of the characteristic triplet of the process $X$ as

$$
a=r-\delta-\sigma^{2} / 2-\int_{\mathbb{R}}\left(e^{y}-1-h(y)\right) \Pi(d y) .
$$

In the case, when

$$
X_{t}=\sigma W_{t}+a t \quad(t \geq 0),
$$

where $W=\left\{W_{t}\right\}_{t \geq 0}$ is a Wiener process, we obtain the Black-ScholesMerton (1973) model (see [3],[23]).

In the market model considered we introduce some derivative assets. More precisely, we consider call and put options, of both European and American types. Denote by $\mathcal{M}_{T}$ the class of stopping times up to a fixed constant time $T$, i.e:

$$
\mathcal{M}_{T}=\{\tau: 0 \leq \tau \leq T, \tau \text { stopping time w.r.t } \mathbf{F}\}
$$

for the finite horizon case and for the perpetual case we take $T=\infty$ and denote by $\overline{\mathcal{M}}$ the resulting stopping times set. Then, for each stopping time $\tau \in \mathcal{M}_{T}$ we introduce

$$
\begin{aligned}
c\left(S_{0}, K, r, \delta, \tau, \psi\right) & =\mathbf{E} e^{-r \tau}\left(S_{\tau}-K\right)^{+}, \\
p\left(S_{0}, K, r, \delta, \tau, \psi\right) & =\mathbf{E} e^{-r \tau}\left(K-S_{\tau}\right)^{+}
\end{aligned}
$$

According to our analysis, (8) and (9) represent auxiliary quantities; additionally they are interesting in their own right as random maturity options, as considered for instance in Schroder (1999) and Detemple (2001). If $\tau=T$, formulas (8) and (9) give the price of the European call and put options respectively. For the American finite case, prices and optimal stopping rules $\tau_{c}^{*}$ and $\tau_{p}^{*}$ are defined, respectively, by:

$$
\begin{aligned}
& C\left(S_{0}, K, r, \delta, T, \psi\right)=\sup _{\tau \in \mathcal{M}_{T}} \mathbf{E} e^{-r \tau}\left(S_{\tau}-K\right)^{+}=\mathbf{E} e^{-r \tau_{c}^{*}}\left(S_{\tau_{c}^{*}}-K\right)^{+} \\
& P\left(S_{0}, K, r, \delta, T, \psi\right)=\sup _{\tau \in \mathcal{M}_{T}} \mathbf{E} e^{-r \tau}\left(K-S_{\tau}\right)^{+}=\mathbf{E} e^{-r \tau_{p}^{*}}\left(K-S_{\tau_{p}^{*}}\right)^{+},
\end{aligned}
$$


and, for the American perpetual case, prices and optimal stopping rules are determined by

$\bar{C}\left(S_{0}, K, r, \delta, \psi\right)=\sup _{\tau \in \mathcal{M}} \mathbf{E} e^{-r \tau}\left(S_{\tau}-K\right)^{+} \mathbf{1}_{\{\tau<\infty\}}=\mathbf{E} e^{-r \tau_{c}^{*}}\left(S_{\tau_{c}^{*}}-K\right)^{+} \mathbf{1}_{\left\{\tau_{c}^{*}<\infty\right\}}$,

$\bar{P}\left(S_{0}, K, r, \delta, \psi\right)=\sup _{\tau \in \overline{\mathcal{M}}} \mathbf{E} e^{-r \tau}\left(K-S_{\tau}\right)^{+} \mathbf{1}_{\{\tau<\infty\}}=\mathbf{E} e^{-r \tau_{p}^{*}}\left(K-S_{\tau_{p}^{*}}\right)^{+} \mathbf{1}_{\left\{\tau_{p}^{*}<\infty\right\}}$.

\subsection{Put Call duality and dual markets}

Lemma 2.1 (Duality). Consider a Lévy market with driving process $X$ with characteristic exponent $\psi(z)$, defined in (3), on the set $\mathbb{C}_{0}$ in (2). Then, for the expectations introduced in (8) and (9) we have

$$
c\left(S_{0}, K, r, \delta, \tau, \psi\right)=p\left(K, S_{0}, \delta, r, \tau, \tilde{\psi}\right),
$$

where

$$
\tilde{\psi}(z)=\tilde{a} z+\frac{1}{2} \tilde{\sigma}^{2} z^{2}+\int_{\mathbb{R}}\left(e^{z y}-1-z h(y)\right) \tilde{\Pi}(d y)
$$

is the characteristic exponent (of a certain Lévy process) that satisfies

$$
\tilde{\psi}(z)=\psi(1-z)-\psi(1), \quad \text { for } 1-z \in \mathbb{C}_{0},
$$

and in consequence,

$$
\begin{cases}\tilde{a} & =\delta-r-\sigma^{2} / 2-\int_{\mathbb{R}}\left(e^{y}-1-h(y)\right) \tilde{\Pi}(d y), \\ \tilde{\sigma} & =\sigma, \\ \tilde{\Pi}(d y) & =e^{-y} \Pi(-d y) .\end{cases}
$$

Remark 2.1. The presented Lemma is very similar to Proposition 1 in Schroder (1999) and the results obtained in [12] and [16]. The main difference is that the particular structure of the underlying process (Lévy process are a particular case of the models considered in [32]) allows to completely characterize the distribution of the dual process $\tilde{X}$ under the dual martingale measure $\tilde{\mathbf{Q}}$, and to give a simpler proof. Considering Additive processes similar result, in the case of European plain vanilla options, were obtained by Eberlein and Papantaleon (2005), see Corollary 4.2 in [12]. 
If we take $\tau=T$ in the Duality Lemma we obtain the following put call relation.

Corollary 2.1 (European Options). For the expectations introduced in (8) and (9) we have

$$
c\left(S_{0}, K, r, \delta, T, \psi\right)=p\left(K, S_{0}, \delta, r, T, \tilde{\psi}\right),
$$

with $\psi$ and $\tilde{\psi}$ as in the Duality Lemma.

To formulate the duality result for American Options, we observe that the optimal stopping rules for the American Call and Put options have, respectively, the form

$$
\begin{aligned}
\tau_{c}^{*} & =\inf \left\{t \geq 0: S_{t} \geq \mathcal{B}_{c}(t)\right\} \wedge T, \\
\tau_{p}^{*} & =\inf \left\{t \geq 0: S_{t} \leq \mathcal{B}_{p}(t)\right\} \wedge T .
\end{aligned}
$$

where the curves $\mathcal{B}_{c}$ and $\mathcal{B}_{p}$ are the boundaries of the continuation region. (See 12.1.3 in Cont and Tankov (2004), or Theorem 6.1 in Boyarchenko and Levendorskii (2002).)

Corollary 2.2 (American Options). For the value functions in (10) and (11) we have

$$
C\left(S_{0}, K, r, \delta, T, \psi\right)=P\left(K, S_{0}, \delta, r, T, \tilde{\psi}\right),
$$

with $\psi$ and $\tilde{\psi}$ as in the Duality Lemma. Furthermore, when $\delta>0$, for the optimal stopping boundaries, we obtain that

$$
\mathcal{B}_{c}(t) \mathcal{B}_{p}(t)=S_{0} K
$$

In case $\delta=0$ we have $\tau_{c}^{*}=\tau_{p}^{*}=T$.

Remark 2.2. The relation between the stopping boundaries is analogous to the one for Itô processes obtained by Detemple (2001) (see equation (30)).

In what respects Perpetual Call and Put American Options, the optimal stopping rules have, respectively, the form

$$
\begin{aligned}
& \tau_{c}^{*}=\inf \left\{t \geq 0: S_{t} \geq S_{c}^{*}\right\}, \\
& \tau_{p}^{*}=\inf \left\{t \geq 0: S_{t} \leq S_{p}^{*}\right\} .
\end{aligned}
$$


where the constants $S_{c}^{*}$ and $S_{p}^{*}$ are the critical prices. (See Theorem 1 and 2 in Mordecki (2002).)

Corollary 2.3 (Perpetual Options). For prices of Perpetual Call and Put options in (12) and (13) we have

$$
\bar{C}\left(S_{0}, K, r, \delta, \psi\right)=\bar{P}\left(K, S_{0}, \delta, r, \tilde{\psi}\right)
$$

with $\psi$ and $\tilde{\psi}$ as in the Duality Lemma. Furthermore, when $\delta>0$, for the optimal stopping levels, we obtain the relation

$$
S_{c}^{*} S_{p}^{*}=S_{0} K
$$

\subsection{Proofs}

Proof of the Duality Lemma. Consider the martingale $Z=\left\{Z_{t}\right\}_{t \geq 0}$ defined by

$$
Z_{t}=e^{X_{t}-(r-\delta) t},(t \geq 0) .
$$

Following Shiryaev et al. [35] we introduce the dual martingale measure $\tilde{\mathbf{Q}}$ given by its restrictions $\tilde{\mathbf{Q}}_{t}$ to $\mathcal{F}_{t}$ by

$$
\frac{d \tilde{\mathbf{Q}}_{t}}{d \mathbf{Q}_{t}}=Z_{t}
$$

where $\mathbf{Q}_{t}$ (resp. $\tilde{\mathbf{Q}}_{t}$ ) is the restriction of $\mathbf{Q}$ (resp. $\tilde{\mathbf{Q}}$ ) to $\mathcal{F}_{t}$. Following (ii) in Theorem III.3.4 in Jacod and Shiryaev (1987), we know that the fixed time can be replaced by any stopping time in $\tau \in \mathcal{M}_{T}$ to give

$$
\frac{d \tilde{\mathbf{Q}}_{\tau}}{d \mathbf{Q}_{\tau}}=Z_{\tau},
$$

where, as stated before, $\mathbf{Q}_{\tau}$ (resp. $\tilde{\mathbf{Q}}_{\tau}$ ) is the restriction of $\mathbf{Q}$ (resp. $\tilde{\mathbf{Q}}$ ) to the $\sigma$-algebra

$$
\mathcal{F}_{\tau}=\left\{A \in \mathcal{F}: A \cap\{\tau \leq t\} \in \mathcal{F}_{t}\right\}
$$

Now

$$
\begin{aligned}
c\left(S_{0}, K, r, \delta, \tau, \psi\right) & =\mathbf{E} e^{-r \tau}\left(S_{0} e^{X_{\tau}}-K\right)^{+}=\mathbf{E} Z_{\tau} e^{-\delta \tau}\left(S_{0}-K e^{-X_{\tau}}\right)^{+} \\
& =\tilde{\mathbf{E}} e^{-\delta \tau}\left(S_{0}-K e^{\tilde{X}_{\tau}}\right)^{+}
\end{aligned}
$$


where the last equality is obtained using the martingale property of $Z$ and the iterated expectations property. Furthermore, $\tilde{\mathbf{E}}$ denotes expectation with respect to $\tilde{\mathbf{Q}}$, and the process $\tilde{X}=\left\{\tilde{X}_{t}\right\}_{t \geq 0}$ given by $\tilde{X}_{t}=-X_{t}(t \geq 0)$ is the dual process (see [2]). In order to conclude the proof, that is, in order to verify that

$$
\tilde{\mathbf{E}} e^{-\delta \tau}\left(S_{0}-K e^{\tilde{X}_{\tau}}\right)^{+}=p\left(K, S_{0}, \delta, r, \tau, \tilde{\psi}\right),
$$

we must verify the dual process $\tilde{X}$ is a Lévy process with characteristic exponent defined by (15) and (16). First, for a complex $z$ such that $1-z \in \mathbb{C}_{0}$, we have

$$
\tilde{\mathbf{E}} e^{z \tilde{X}_{t}}=\mathbf{E} Z_{t} e^{-z X_{t}}=\mathbf{E} e^{-(r-\delta) t} e^{X_{t}} e^{-z X_{t}}=e^{t(\psi(1-z)-\psi(1))} .
$$

Second, defining $\tilde{\psi}(z)=\psi(1-z)-\psi(1)$, we have

$$
\begin{aligned}
\tilde{\psi}(z)= & a(1-z)+\sigma^{2}(1-z)^{2} / 2+\int_{\mathbb{R}}\left(e^{(1-z) y}-1-(1-z) h(y)\right) \Pi(d y) \\
& -a-\frac{1}{2} \sigma^{2}-\int_{\mathbb{R}}\left(e^{y}-1-h(y)\right) \Pi(d y) \\
= & -\left(a+\sigma^{2}\right) z+\frac{1}{2} \sigma^{2} z^{2}+\int_{\mathbb{R}}\left(e^{(1-z) y}-e^{y}+z h(y)\right) \Pi(d y) .
\end{aligned}
$$

The integral term is transformed as follows:

$$
\begin{aligned}
& \int_{\mathbb{R}}\left(e^{(1-z) y}-e^{y}+z h(y)\right) \Pi(d y) \\
&=z \int_{\mathbb{R}}\left(1-e^{y}\right) h(y) \Pi(d y)+\int_{\mathbb{R}}\left(e^{-z y}-1+z h(y)\right) e^{y} \Pi(d y) \\
&=z \int_{\mathbb{R}}\left(1-e^{y}\right) h(y) \Pi(d y)+\int_{\mathbb{R}}\left(e^{z y}-1-z h(y)\right) \tilde{\Pi}(d y)
\end{aligned}
$$

where we introduced the change of variables $y=-u$ in the last integral, and denoted $\tilde{\Pi}(d y)=e^{-y} \Pi(-d y)$. The final calculation, taking into account (6), 
is

$$
\begin{aligned}
-\tilde{a} & =a+\sigma^{2} / 2+\int_{\mathbb{R}}\left(e^{y}-1\right) h(y) \Pi(d y) \\
& =r-\delta-\sigma^{2} / 2-\int_{\mathbb{R}}\left(e^{y}-1-h(y)\right) \Pi(d y)+\sigma^{2} / 2+\int_{\mathbb{R}}\left(e^{y}-1\right) h(y) \Pi(d y) \\
& =r-\delta+\sigma^{2} / 2+\int_{\mathbb{R}}\left(e^{y}-1-h(y)\right) \tilde{\Pi}(d y) .
\end{aligned}
$$

This concludes the proof.

Proof of Corollary 2.2 (American Options). Formula (18) is obtained taking the supremum over all $\tau \in \mathcal{M}_{T}$ in equation (14). Let us see then (19). Assume first that $\delta=0$. Then, Merton's observation about the optimality of American Call options without dividends apply, in consequence prices of American Call and European options coincide with $\tau_{c}^{*}=T$. By the Duality Lemma (or by the same reasoning with put options when $r=0$ ) we obtain that $\tau_{p}^{*}=T$.

Assume now $\delta>0$. To prove (19), we apply the Duality Lemma for the optimal stopping time $\tau_{c}^{*}$ :

$$
\begin{aligned}
C\left(S_{0}, K, r, \delta, T, \psi\right) & =c\left(S_{0}, K, r, \delta, \tau_{c}^{*}, \psi\right)=p\left(K, S_{0}, \delta, r, \tau_{c}^{*}, \psi\right) \\
& =P\left(K, S_{0}, \delta, r, T, \psi\right),
\end{aligned}
$$

where the last equality follows from the fact that for each stopping time we obtain a call price equal to the corresponding put price, and $\tau_{c}^{*}$ gives the supremum. We express the stopping time $\tau_{c}^{*}$ in terms of $\tilde{X}$, the dual process:

$$
\tau_{c}^{*}=\inf \left\{t \geq 0: S_{0} e^{X_{t}} \geq \mathcal{B}_{c}(t)\right\} \wedge T=\inf \left\{t \geq 0: K e^{-X_{t}} \leq \frac{K S_{0}}{\mathcal{B}_{c}(t)}\right\} \wedge T .
$$

As, on the other side

$$
\tau_{p}^{*}=\inf \left\{t \geq 0: S_{t} \leq \mathcal{B}_{p}(t)\right\} \wedge T,
$$

and the stopping region is unique, we obtain the identity (19).

Proof of Corollary 2.3 (Perpetual Options). We have to distinguish between cases $\delta>0$ and $\delta=0$. (a) Assume first that $\delta>0$. We claim that

$$
\lim _{t \rightarrow \infty} X_{t}-r t=-\infty
$$


As $1+x \leq e^{x}$, and the Lévy process does not degenerate, the claim follows from

$$
\mathbf{E}\left(X_{1}-r\right)<\mathbf{E} e^{X_{1}-r}-1=e^{\psi(1)-r}-1=e^{-\delta}-1<0,
$$

and the knowledge of the asymptotic behaviour of a Lévy process with negative expectation, obtained by Rogozin (1966). Based on our claim, for an arbitrary stopping time $\tau$, possibly taking the value $\infty$, when $T \rightarrow \infty$, we have

$$
\mathbf{E} e^{-r(\tau \wedge T)}\left(S_{\tau \wedge T}-K\right)^{+} \rightarrow \mathbf{E} e^{-r \tau}\left(S_{\tau}-K\right)^{+} \mathbf{1}_{\{\tau<\infty\}} .
$$

Introduce

$$
M_{r}=\sup _{0 \leq t<\tau(r)} X_{t} \quad \text { and } \quad I_{r}=\inf _{0 \leq t<\tau(r)} X_{t},
$$

where $\tau(r)$ is an exponential random variable with parameter $r>0$, independent of $X$, and $\tau(0)=\infty$. For the optimal stopping rule ([25]), given by

$$
\tau_{c}^{*}=\left\{t \geq 0: S_{t} \geq S_{c}^{*}\right\},
$$

with $S_{c}^{*}=K \mathbf{E} e^{M_{r}}$, we know that

$$
\begin{aligned}
\mathbf{E} e^{-r\left(\tau_{c}^{*} \wedge T\right)}\left(S_{\tau_{c}^{*} \wedge T}-K\right)^{+} & \leq \sup _{\tau \in \mathcal{M}_{T}} \mathbf{E} e^{-r \tau}\left(S_{\tau}-K\right)^{+} \\
& \leq \sup _{\tau \in \overline{\mathcal{M}}} \mathbf{E} e^{-r \tau}\left(S_{\tau}-K\right)^{+} \mathbf{1}_{\{\tau<\infty\}} .
\end{aligned}
$$

From this, taking into account (22), we obtain that

$$
\lim _{T \rightarrow \infty} C\left(S_{0}, K, r, \delta, T, \psi\right)=\bar{C}\left(S_{0}, K, r, \delta, \psi\right) .
$$

The argument for the Put perpetual option is similar, giving

$$
\lim _{T \rightarrow \infty} P\left(K, S_{0}, \delta, r, T, \tilde{\psi}\right)=\bar{P}\left(K, S_{0}, \delta, r, \tilde{\psi}\right) .
$$

The equality in (20) then follows taking limit as value $T \rightarrow \infty$ in (18).

(b) Assume now $\delta=0$. By Merton's observation, we have

$$
\sup _{\tau \in \mathcal{M}_{T}} \mathbf{E} e^{-r \tau}\left(S_{\tau}-K\right)^{+}=\mathbf{E} e^{-r T}\left(S_{T}-K\right)^{+},
$$


similarly for the Put case, and the conclusion follows as before.

Let us see (21). For the perpetual put, on the dual market, the critical level is given by

$$
S_{p}^{*}=S_{0} \tilde{\mathbf{E}} e^{\tilde{I_{\delta}}}
$$

as follows from Theorem 2 in [25]. We express this quantity in the risk neutral market:

$$
\begin{aligned}
\tilde{\mathbf{E}} e^{\tilde{I_{\delta}}} & =\int_{0}^{\infty} \tilde{\mathbf{E}} e^{\inf _{0 \leq s \leq t}\left(-X_{s}\right)} \delta e^{-\delta t} d t=\int_{0}^{\infty} \mathbf{E}\left(e^{-(r-\delta) t} e^{X_{t}-\sup _{0 \leq s \leq t} X_{s}}\right) \delta e^{-\delta t} d t \\
& =\frac{\delta}{r} \int_{0}^{\infty} \mathbf{E}\left(e^{X_{t}-\sup _{0 \leq s \leq t} X_{s}}\right) r e^{-r t} d t \\
& =\frac{\delta}{r} \mathbf{E} e^{X_{\tau(r)}-M_{r}}=\frac{\delta}{r} \mathbf{E} e^{I_{r}} .
\end{aligned}
$$

Here we used the change of probability measure from the dual martingale measure, and, in the last line, a fluctuation identity (Section VI.2 in Bertoin (1996)). Now, applying the Wiener-Hopf equation due to Rogozin (1966), we have

$$
S_{c}^{*} S_{p}^{*}=\left(K \mathbf{E} e^{M_{r}}\right)\left(S_{0} \frac{\delta}{r} \mathbf{E} e^{I_{r}}\right)=K S_{0} \frac{\delta}{r-\psi(1)}=K S_{0},
$$

because $r-\psi(1)=\delta$. This concludes the proof.

\subsection{Dual Markets}

The Duality Lemma motivates us to introduce the following market model. Given a Lévy market with driving process characterized by $\psi$ in (3), consider a market model with two assets, a deterministic savings account $\tilde{B}=\left\{\tilde{B}_{t}\right\}_{t \geq 0}$, given by

$$
\tilde{B}_{t}=e^{\delta t}, \quad \delta \geq 0,
$$

and a stock $\tilde{S}=\left\{\widetilde{S}_{t}\right\}_{t \geq 0}$, modelled by

$$
\tilde{S}_{t}=K e^{\tilde{X}_{t}}, \quad \tilde{S}_{0}=K>0,
$$

where $\tilde{X}=\left\{\tilde{X}_{t}\right\}_{t \geq 0}$ is a Lévy process with characteristic exponent under $\tilde{\mathbf{Q}}$ given by $\tilde{\psi}$ in (15). The process $\tilde{S}_{t}$ represents the price of $K S_{0}$ dollars measured in units of stock $S$. This market is the auxiliary market in Detemple 
(2001), and we call it dual market; accordingly, we call Put-Call duality the relation (14). It must be noticed that Peskir and Shiryaev (2001) propose the same denomination for a different relation in [26]. Finally, observe that in the dual market (i.e. with respect to $\tilde{\mathbf{Q}})$ the process $\left\{e^{-(\delta-r) t} \tilde{S}_{t}\right\}$ is a martingale. As a consequence, we obtain the Put-Call symmetry in the Black-ScholesMerton model: In this case $\Pi=0$, we have no jumps, and the characteristic exponents are

$$
\begin{aligned}
& \psi(z)=\left(r-\delta-\sigma^{2} / 2\right) z+\sigma^{2} z^{2} / 2, \\
& \tilde{\psi}(z)=\left(\delta-r-\sigma^{2} / 2\right) z+\sigma^{2} z^{2} / 2 .
\end{aligned}
$$

and relation (14) is the result known as put-call symmetry. In the presence of jumps like the jump-diffusion model of Merton (1976), if the jump returns of $S$ under $\mathbf{Q}$ and $\tilde{S}$ under $\tilde{\mathbf{Q}}$ have the same distribution, the Duality Lemma implies that by exchanging the roles of $\delta$ by $r$ and $K$ by $S_{0}$ in (14) and (16), we can obtain an American call price formula from the American put price formula. Motivated by this analysis we introduce the definition of symmetric markets in the following section.

\section{Market Symmetry}

It is interesting to note that in a market with no jumps (i.e. in the BlackScholes model), the distribution of the discounted and reinvested stock both in the given risk neutral and in the dual Lévy market, taking equal initial values, coincide. It is then natural to define a Lévy market to be symmetric when this relation holds, i.e. when

$$
\mathcal{L}\left(e^{X_{t}-(r-\delta) t} \mid \mathbf{Q}\right)=\mathcal{L}\left(e^{-X_{t}-(\delta-r) t} \mid \tilde{\mathbf{Q}}\right),
$$

meaning equality in law. In view of (16), and due to the fact that the characteristic triplet determines the law of a Lévy processes, we obtain that a necessary and sufficient condition for (24) to hold is

$$
\Pi(d y)=e^{-y} \Pi(-d y) .
$$

This ensures $\tilde{\Pi}=\Pi$, and from this follows $a-(r-\delta)=\tilde{a}-(\delta-r)$, giving (24), as we always have $\tilde{\sigma}=\sigma$. Condition (25) answers a question raised by Carr and Chesney (1996), see [5]. 


\subsection{Diffusions with jumps}

Consider the jump - diffusion model proposed by Merton (1976) in [24]. The driving Lévy process in this model has Lévy measure given by

$$
\Pi(d y)=\lambda \frac{1}{\delta \sqrt{2 \pi}} e^{-(y-\mu)^{2} /\left(2 \delta^{2}\right)} d y,
$$

it is easy to verify that condition (25) holds if and only if $2 \mu+\delta^{2}=0$. This result was obtained by Bates (1997) in [1] for future options, that result is obtained as a particular case, if we replace the future price as being the underlying asset, when $r=\delta$.

\subsection{Bates's $x \%$-Rule}

If $r=\delta$ we can take the future price $F$ as the underlying asset in Corollary (2.1). Then, we can obtain the Bate's $x \%$ rule in a more general Lévy market.

Corollary 3.1. Assume (25) holds (or in the particular case (31), assume $\beta=-1 / 2)$, we have

$$
c\left(F, K_{c}, r, \tau, \psi\right)=x p\left(F, K_{p}, r, \tau, \psi\right),
$$

where $K_{c}=x F$ and $K_{p}=F / x$, with $x>0$.

From here, calls and puts at-the-money should have the same price. That is, if the call and put options have strike prices $x \%$ out-of-the money relative to the future price, then the call should be priced $x \%$ higher than the put.

Remark 3.1 (Implied volatility). Any model satisfying (25) must have identical Black-Scholes implicit volatilities for calls and puts with strikes $\ln \left(K_{c} / F\right)=\ln x=-\ln \left(K_{p} / F\right)$, where $x>0$ is arbitrary. That is, the volatility smile curve is symmetric in the moneyness $\ln (K / F)$. Remember that prices satisfy (26) and by put-call parity, European calls and puts with same strike and maturity must have identical implicit volatilities.

\section{Asymmetry in Lévy markets}

Our intention is to review several concrete models proposed in the literature. We restrict ourselves to Lévy markets with jump measures of the form 


$$
\Pi(d y)=e^{\beta y} \Pi_{0}(d y),
$$

where $\Pi_{0}(d y)$ is a symmetric measure, i.e. $\Pi_{0}(d y)=\Pi_{0}(-d y)$, everything with respect to the risk neutral measure $\mathbf{Q}$.

As a consequence of (25), we obtain that the market is symmetric if and only if $\beta=-1 / 2$. In view of this, we propose to measure the asymmetry in the market through the parameter $\beta+1 / 2$. When $\beta+1 / 2=0$, we have a symmetric market.

Our proposal is similar, in certain sense, to the skewness premium introduced by Bates (1997) in [1]. That is, when the market is symmetric, the skewness premium is obtained using the $x \%$-rule. The idea is to describe numerically the departure from the symmetry, the main difference with Bates (1997) is that the parameter $\beta$ is a property of the market, independent of the derivative asset considered.

Although from a theoretical point of view the assumption (27) is a real restriction, most models in practice share this property, and furthermore, they have a jump measure that has a Radon-Nikodym density. In this case, we have

$$
\Pi(d y)=e^{\beta y} p(y) d y,
$$

where $p(y)=p(-y)$, i.e. the function $p(y)$ is even. See Kou and Wang (2004) in case $p=q=1 / 2$, and the examples below, in 4.1.1, 4.1.2 and 4.2.1.

More precisely, many parametric models that we found in the literature, in what concerns Lévy markets, including diffusions with jumps, can be reparametrized in the form (28). As we will see, empirical risk-neutral markets are not symmetric, and in view of (28), we propose to model the asymmetry of the market through the parameter $\beta+1 / 2$. Before considering concrete examples, we review the Esscher transform.

\subsection{Esscher transform and asymmetry}

We now review some notation and useful facts. All the developments up to now where with respect to the risk neutral martingale measure $\mathbf{Q}$. Now we consider that there is an historical probability measure $\mathbf{P}$ and that $\mathbf{Q}$ is the consequence of an Esscher transform. It is clear that this is one between several possibilities, and we refer to Chan (1999) and Shiryaev (1999) for a discussion on this topic. In consequence, when necessary, we add a subscript 
$\mathbf{P}$ to refer to parameters under the historical probability measure $\mathbf{P}$, i.e. $\psi_{\mathbf{P}}$, $\Pi_{\mathbf{P}}$, and even sometimes we use the subscript $\mathbf{Q}$ to distinguish risk neutral parameters. As we said, the link between $\mathbf{P}$ and $\mathbf{Q}$ is given by the Esscher transform, and this is stated through the change of measure

$$
d \mathbf{Q}_{t}=e^{\theta X_{t}-t \psi_{\mathbf{P}}(\theta)} d \mathbf{P}_{t}
$$

where $\theta$ is a parameter to be determined. From (29) follows that

$$
\psi(z)=\psi_{\mathbf{Q}}(z)=\psi_{\mathbf{P}}(z+\theta)-\psi_{\mathbf{P}}(z),
$$

as we require that the discounted and reinvested stock is a martingale under Q, i.e. $\left\{e^{-(r-\delta) t} S_{t}\right\}$ is a $\mathbf{Q}$-martingale, we obtain that

$$
\psi(1)=\psi_{\mathbf{P}}(1+\theta)-\psi_{\mathbf{P}}(1)=r-\delta,
$$

and this determines $\theta$. It is relevant for us, that from (30) follows

$$
\Pi_{\mathbf{Q}}(d y)=e^{\theta y} \Pi_{\mathbf{P}}(d y) .
$$

(See Theorem VII.3.2 in Shiryaev (1999).) If we combine this result with our model assumption (27) we conclude that

$$
e^{\beta y} \Pi_{0}(d y)=e^{\theta y} \Pi_{\mathbf{P}}(d y),
$$

meaning that the form of the jump measure under $\mathbf{P}$ is

$$
\Pi_{\mathbf{P}}(d y)=e^{(\beta-\theta) y} \Pi_{0}(d y)=e^{\beta_{\mathbf{P}} y} \Pi_{0}(d y),
$$

that is, the same form with a translated parameter. We conclude, that under the Esscher transform, our model assumption (27) is equivalent to the assumption (31), and that the relation between the symmetry parameters is

$$
\beta=\beta_{\mathbf{Q}}=\beta_{\mathbf{P}}+\theta .
$$

\subsubsection{The Generalized Hyperbolic Model}

This model has been proposed by Eberlein and Prause (2002), these distributions allow for a more realistic description of asset returns (see [13] and [11]). This model, under $\mathbf{P}$, has $\sigma=0$, and a Lévy measure given by (31), with 


$$
p(y)=\frac{1}{|y|}\left(\int_{0}^{\infty} \frac{\exp \left(-\sqrt{2 z+\alpha^{2}}|y|\right)}{\pi^{2} z\left(J_{\lambda}^{2}(\delta \sqrt{2 z})+Y_{\lambda}^{2}(\delta \sqrt{2 z})\right)} d z+\mathbf{1}_{\{\lambda \geq 0\}} \lambda e^{-\alpha|y|}\right),
$$

where $\alpha, \beta_{\mathbf{P}}, \lambda, \delta$ are the historical parameters that satisfy the conditions $0 \leq\left|\beta_{\mathbf{P}}\right|<\alpha$, and $\delta>0$; and $J_{\lambda}, Y_{\lambda}$ are the Bessel functions of the first and second kind (for details see [13]). Particular cases are the hyperbolic distribution, obtained when $\lambda=1$; and the normal inverse gaussian (NIG) when $\lambda=-1 / 2$.

Using daily returns from Brazilian Index Ibovespa for the period 07/01/1994 to 12/13/2001, Fajardo and Farias (2004) estimate the parameter $\beta_{\mathbf{P}}=-0.0035$ for the NIG distribution and the estimate $\beta_{\mathbf{Q}}=80.65$ for the risk neutral distribution, given by (32). They also estimate the parameters for various Brazilian assets finding $\beta_{\mathbf{Q}} \neq-1 / 2$. This indicates absence of symmetry.

\subsubsection{The Meixner Model}

The Meixner process was proposed to model financial data by Grigelionis (1999) in [19] and by Schoutens (2002) in [30]. The Lévy process derived from this distribution has, under $\mathbf{P}$, the following Lévy measure:

$$
\Pi(y)=c \frac{e^{\frac{b}{a} y}}{y \sinh (\pi y / a)} d y
$$

where $a, b$ and $c$ are parameters of the Meixner density, such that $a>0$, $-\pi<b<\pi$ and $c>0$. The Lévy measure also corresponds to one of the form (31), if we take $\beta_{\mathbf{P}}=b / a$, and

$$
p(y)=\frac{c}{y \sinh (\pi y / a)} .
$$

Using daily returns from various index, Schoutens in [29] found parameters estimates $\hat{a}$ and $\hat{b}$ for the period $1 / 1 / 1997$ to $31 / 12 / 1999$. We summarize these results and the corresponding parameter $\beta_{\mathbf{Q}}=\hat{b} / \hat{a}+\theta$ in Table 1 .

\subsection{Market Risk Neutral Measure}

In order to see what happens if we use another risk neutral measure different from the given by the Esscher transform, we give the following example, 


\begin{tabular}{|c|c|c|c|c|}
\hline Index & $\hat{a}$ & $\hat{b}$ & $\theta$ & $\beta_{\mathbf{Q}}+1 / 2$ \\
\hline Nikkei 225 & 0.02982825 & 0.12716244 & 0.42190524 & 5.18506 \\
DAX & 0.02612297 & -0.50801886 & -4.46513538 & -23.4123 \\
FTSE-100 & 0.01502403 & -0.014336370 & -4.34746821 & -4.8017 \\
SMI & 0.02954687 & -0.33888717 & -3.97213216 & -14.9416 \\
Nasdaq Comp. & 0.03346698 & -0.49356259 & -5.95888693 & -20.2066 \\
CAC-40. & 0.02539854 & -0.23804755 & -5.77928595 & -14.6518 \\
\hline
\end{tabular}

Table 1: Estimates of the Meixner Distribution

where the risk neutral measure used is the measure obtained from the observed option market prices.

\subsubsection{The CGMY model}

This Lévy market model, proposed by Carr et al. (2002) in [7] is characterized by $\sigma=0$ and Lévy measure given by (31), where the function $p(y)$ is given by

$$
p(y)=\frac{C}{|y|^{1+Y}} e^{-\alpha|y|} .
$$

The parameters satisfy $C>0, Y<2$, and $G=\alpha+\beta \geq 0, M=\alpha-\beta \geq 0$, where $C, G, M, Y$ are the parameters used in [7].

For studying the presence of a pure diffusion component in the model, condition $\sigma=0$ is relaxed, and risk neutral distribution is estimated in a five parameters model. Values of $\beta=(G-M) / 2$ are given for different assets in Table 3 in [7], and in the general situation, the parameter $\beta$ is negative, and less than $-1 / 2$. The condition needed in this case for the market to be symmetric is $G=M-1$. It is worth noting that the finite moment log-stable (LS) model of Carr and $\mathrm{Wu}$ (2003) can be regarded as a limit case of the CGMY model with $G=0$ (no exponential factor) and $M=\infty$ (no positive jumps). This market model is not symmetric.

This suggest the use of the risk neutral asymmetry parameter $\beta$, obtained from prices as a test to know whether the popular Esscher transform used in Lévy models is correct. 


\section{Conclusions}

Departing from duality, a relation between call and put prices, obtained through a change of numeraire, and corresponding to a change of probability measure in a Lévy market model under a given risk neutral probability measure, the main contribution of this paper is the characterization of symmetry in these market models, a notion that is also introduced. Symmetric markets have symmetric volatilities smile curves in the log of the ratio strike/futures prices.

This characterization allows to introduce a parameter in the risk neutral model that, in certain sense, measures the asymmetry of a Lévy market model. We also find the expression of this asymmetry parameter in the historical market model, assuming that we rely in the Esscher transform to obtain the given risk neutral measure. We analyze popular models used in the literature, concluding that, in general, markets are not symmetric. Further research is (i) a more detailed empirical investigation of the asymmetry parameter in several models, (ii) the study of the relation between call and put option prices depending on this parameter, and finally, (iii) a conclusion about the opportunity of using Esscher transform, as it induces certain asymmetry relation that can be different from the one observed in option prices.

\section{References}

[1] Bates, D. (1997): "The skewness premium: Option Pricing under Asymmetric Processes". Advances in Futures and Options Research, 9, $51-82$.

[2] Bertoin J. (1996): Lévy Processes. Cambridge University Press, Cambridge.

[3] Black, F. And Scholes, M. (1973): "The pricing of options and corporate liabilities". Journal of Political Economy 81, 637-659.

[4] Boyarchenko, S.I. And Levendorskit, S.Z. (2002): Non-Gaussian Merton-Black-Scholes theory. Advanced Series on Statistical Science \& Applied Probability. 9. Singapore: World Scientific. 
[5] Carr, P. And Chesney, M. (1996): "American Put Call Symmetry". Preprint.

[6] Carr, P. And Wu, L. (2003): "Finite Moment Log Stable Process and Option Pricing". Journal of Finance 58, 753-777.

[7] Carr, P., Geman, H., Madan, D.B. and Yor, M. (2002): "The Fine Structure of Assets Returns: An Empirical Investigation". Journal of Business, Vol 75, No. 2, 305-332.

[8] Chan, T. (1999): "Pricing Contingent Claims on Stocks Driven by Lévy Processes". Annals of Applied Probability, Vol. 9, No. 2, 504-528.

[9] Cont, R. and Tankov, P. (2004): Financial modelling with jump processes., Chapman \& Hall /CRC Financial Mathematics Series.

[10] Detemple, J. (2001): "American options: symmetry properties". In: Option pricing, interest rates and risk management Eds. Jouini, Cvitanic, Musiela. Cambridge University Press, 67-104.

[11] Eberlein, E., Keller, U. And Prause, K. (1998): "New insights into smile, mispricing and value at risk: the hyperbolic model". Journal of Business 71, 371-405.

[12] Eberlein, E. And Papapantoleon, A. (2005): "Symmetries and pricing of exotic options in Lévy models". In Exotic option pricing and advanced Lévy models, A. Kyprianou, W. Schoutens, P. Wilmott (Eds.), Wiley, 99-128.

[13] Eberlein, E. And Prause, K. (2002): "The generalized hyperbolic model: financial derivatives and risk measures". In Mathematical Finance-Bachelier Congress 2000, H. Geman, D. Madan, S. Pliska, T. Vorst (Eds.), Springer Verlag, 245-267.

[14] Fajardo, J. and Farias, A. (2004): "Generalized Hyperbolic Distributions and Brazilian Data". Brazilian Review of Econometrics, Vol. 24, No. 2, 249-271.

[15] Fajardo, J. And Mordecki, E. (2003): "Duality and derivative pricing with Lévy processes." Prepublicaciones de la Unversidad de la República, 2003/76. 
[16] Fajardo, J. And Mordecki, E. (2005): "Duality and Derivative Pricing with Time-Changed Lévy processes". Working paper IBMEC.

[17] H. Geman, N. El Karoui and J.-C. Rochet, (1995): "Changes of numéraire, changes of probability measure and option pricing". J. Appl. Probab, Vol. 32, No. 2, 443-458.

[18] Grabbe, O. (1983): "The Pricing of Call and Put Options on Foreign Exchange". Journal of International Money and Finance, 2, 239-253.

[19] Grigelionis, B. (1999): "Processes of Meixner Type". Lith. Math. J., Vol. 39, No. 1, 33-41.

[20] Jacod, J. And Shiryaev, A.N. (1987): Limit Theorems for Stochastic Processes. Springer, Berlin, Heidelberg.

[21] Kou, S.G. And Wang, H. (2004): "Option pricing Under a Double Exponential Jump Diffusion Model". Manag. Sci., Vol. 50, No. 9, 1178-1192.

[22] Kramkov, D.O. and Mordecki, E. (1994): "Integral option", Theory Probab. Appl., Vol 39, No. 1, 162-172.

[23] Merton, R.C. (1973): "Theory of rational option pricing". Bell J. Econom. Manag. Sci., 4, 141-183.

[24] Merton, R.C. (1976): "Option pricing when the underlying stock returns are discontinuous". J. of Financial Economics, 3, 125-144.

[25] Mordecki, E. (2002): "Optimal stopping and perpetual options for Lévy processes.", Finance Stoch., Vol. 6, No. 4, 473-493.

[26] Peskir, G. And Shiryaev, A. N. (2001): "A note on the call-put parity and a call-put duality". Theory Probab. Appl., 46, 181-183.

[27] Rogozin, B.A. (1966): "On distributions of functionals related to boundary problems for processes with independent increments." Theory of Probability and its Applications Vol. XI, No. 4, 580-591.

[28] Sato, K. -I. (1999): Lévy processes and infinitely divisible distributions. Cambridge Studies in Advanced Mathematics, 68. Cambridge University Press, Cambridge. 
[29] Schoutens, W. (2001): "The Meixner Process in Finance". EURANDOM Report 2001-002, EURANDOM, Eindhoven.

[30] Schoutens, W. (2002): "The Meixner process: Theory and Applications in Finance". In Mini-proceedings of the 2nd MathPhysto Conference on Lévy processes. O. E. Barndorff-Nielsen (Ed.), 237-241.

[31] Schoutens, W. (2003): Lévy Processes in Finance, Wiley Series in Probability and Statistics.

[32] Schroder, M. (1999): "Change of Numeraire for Pricing Futures, Forwards, and Options". The Review of Financial Studies, Vol. 12, No. 5, 1143-1163.

[33] Shepp L.A. And Shiryaev A.N. (1994): "New look at the pricing of Russian option", Theory of Probability and Applications Vol 39, No. 1, 103-119.

[34] Shiryaev, A. N. (1999): Essentials of stochastic finance: facts, models, theory. Singapore: World Scientic.

[35] Shiryaev, A.N., Kabanov, Y. M., Kramkov, D.O. and MelNIKOV, A.V. (1994): "On the pricing of options of European and American types, II. Continuous time". Theory of Probability and Applications, 39, 61-102.

[36] Skorokhod, A. V. (1991). Random processes with independent increments. Kluwer Academic Publishers, Dordrecht. 